EPJ Web of Conferences 78, 03004 (2014)

DOI: 10.1051 /epjconf / 20147803004

(C) Owned by the authors, published by EDP Sciences, 2014

\title{
Geometry of shell-model matrix elements
}

\author{
P. Van Isacker ${ }^{\mathrm{a}}$ \\ GANIL, CEA/DSM-CNRS/IN2P3, B.P. 55027, F-14076 Caen Cedex 5, France
}

\begin{abstract}
With use of Wigner's classical-limit expressions for $3 j$ symbols, matrix elements of the nuclear shell model are given a geometric interpretation in terms of the angle between neutron and proton angular momenta.
\end{abstract}

\section{Introduction}

In present-day applications of the nuclear shell model it is customary to consider an effective nucleonnucleon interaction appropriate for a Hilbert space of huge dimension [1]. Although this approach generally is successful, it is computationally intensive and additional intuitive understanding of the numerical results is called for. A classic example of the latter approach was given by Schiffer [2] who pointed out the universal behaviour of the effective interaction in terms of the angle between the angular momenta of the interacting nucleons, property which was later shown to be related to the short-range character of the effective interaction [3]. This geometric interpretation of the nucleonnucleon interaction has become standard knowledge and can indeed be found in many a textbook on nuclear structure, e.g. [4].

It is the purpose of this contribution to show that geometric insight can be extended to more complex shell-model configurations. The general expression for a matrix element, given in section 2, is first applied to the known case of 1 neutron and 1 proton (section 3), and subsequently to the new case of 2 neutrons and 2 protons (section 4 ).

\section{General expression for a neutron-proton matrix element}

Consider a shell-model configuration with $m$ neutrons in the orbits $j_{1 v}, j_{2 v}, \ldots, j_{m v}$, and $m^{\prime}$ protons in the orbits $j_{1 \pi}, j_{2 \pi}, \ldots, j_{m^{\prime} \pi}$, where $j_{k \rho}(\rho=v, \pi)$ is used as an abbreviation for the set of labels $n_{k \rho}$ (radial quantum number), $\ell_{k \rho}$ (orbital angular momentum) and $j_{k \rho}$ (total angular momentum) of singe-particle levels in a central potential. The $m$-neutron- $m^{\prime}$-proton states are denoted as $\left|J_{v}\right\rangle \equiv$ $\left|j_{1 v} j_{2 v}\left(J_{12 v}\right) \ldots J_{v}\right\rangle$ and $\left|J_{\pi}\right\rangle \equiv\left|j_{1 \pi} j_{2 \pi}\left(J_{12 \pi}\right) \ldots J_{\pi}\right\rangle$, respectively, where $J_{12 \ldots \rho}$ are intermediate angular momenta occurring in some coupling scheme, and $J_{v}$ and $J_{\pi}$ are the neutron and proton total angular momenta. The single-particle orbits $j_{k \rho}(k=1,2, \ldots)$ may or may not be identical but in all cases the states $\left|J_{v}\right\rangle$ and $\left|J_{\pi}\right\rangle$ are assumed to be Pauli allowed. It is also of interest to consider the case of hole-like neutrons or protons which will be denoted as $\left|J_{v}^{-1}\right\rangle$ or $\left|J_{\pi}^{-1}\right\rangle$.

A general shell-model hamiltonian has a neutron and a proton piece, and a neutron-proton interaction, and therefore can be written as $\hat{H}=\hat{H}_{v}+\hat{H}_{\pi}+\hat{V}_{v \pi}$. An expression can be derived for the diagonal

\footnotetext{
a e-mail: isacker@ganil.fr
} 
matrix element of this hamiltonian between neutron-proton coupled states $\left|J_{v} J_{\pi} ; J\right\rangle$, assuming fixed configurations $\left|J_{v}\right\rangle$ and $\left|J_{\pi}\right\rangle$, which are coupled to total angular momentum $J$, varying between $\left|J_{v}-J_{\pi}\right|$ and $J_{v}+J_{\pi}$. The aim is to obtain a geometric interpretation of this matrix element as a function of $J_{v}$, $J_{\pi}$ and $J$.

Due to the scalar character of the hamiltonian, the matrix elements of $\hat{H}_{v}$ and $\hat{H}_{\pi}$ depend only on the quantum numbers appearing in $\left|J_{v}\right\rangle$ and $\left|J_{\pi}\right\rangle$, respectively; $\hat{H}_{v}$ and $\hat{H}_{\pi}$ give constant energy contributions, independent of the total angular momentum $J$, and are therefore of no interest in the following. Any $J$ dependence originates from the neutron-proton interaction $\hat{V}_{v \pi}$, specified by its matrix elements $\left\langle j_{k v} j_{l \pi} ; R\left|\hat{V}_{v \pi}\right| j_{k^{\prime} v} j_{l^{\prime} \pi} ; R\right\rangle$. A multipole expansion of $\hat{V}_{v \pi}$ leads to the following expression for the matrix element:

$$
\begin{aligned}
& \left\langle J_{v} J_{\pi} ; J\left|\hat{V}_{v \pi}\right| J_{v} J_{\pi} ; J\right\rangle \\
& =\sum_{k l R \lambda}(-)^{j_{k v}+j_{l \pi}+J_{v}+J_{\pi}+J+R}(2 R+1) V_{j_{k v} j_{l \pi}}^{R}\left\{\begin{array}{ccc}
j_{k v} & j_{k v} & \lambda \\
j_{l \pi} & j_{l \pi} & R
\end{array}\right\}\left\{\begin{array}{ccc}
J_{v} & J_{\pi} & J \\
J_{\pi} & J_{v} & \lambda
\end{array}\right\} \\
& \quad \times\left\langle J_{v}\left\|\left(a_{j_{k v}}^{\dagger} \tilde{a}_{j_{k v}}\right)^{(\lambda)}\right\| J_{v}\right\rangle\left\langle J_{\pi}\left\|\left(a_{j_{l \pi}}^{\dagger} \tilde{a}_{j_{l \pi}}\right)^{(\lambda)}\right\| J_{\pi}\right\rangle,
\end{aligned}
$$

where $V_{j_{k v}}^{R} j_{l \pi} \equiv\left\langle j_{k v} j_{l \pi} ; R\left|\hat{V}_{v \pi}\right| j_{k v} j_{l \pi} ; R\right\rangle$. The operator $a_{j_{k \rho} m_{k \rho}}^{\dagger}$ creates a neutron $(\rho=v)$ or a proton $(\rho=\pi)$ in orbit $j_{k \rho}$ with projection $m_{k \rho}$ on the $z$ axis; the corresponding annihilation operator $a_{j_{k \rho} m_{k \rho}}$ is modified to $\tilde{a}_{j_{k \rho} m_{k \rho}} \equiv(-)^{j_{k \rho}+m_{k \rho}} a_{j_{k \rho}-m_{k \rho}}$ to make it behave as a proper tensor under rotations in three dimensions.

The expression (1) between $m$-neutron- $m^{\prime}$-proton states can be further reduced if a surface delta interaction (SDI) is considered,

$$
\hat{V}^{\mathrm{SDI}}=\sum_{k<l} \hat{V}^{\mathrm{SDI}}(k, l), \quad \hat{V}^{\mathrm{SDI}}(k, l)=-4 \pi a_{T}^{\prime} \delta\left(\bar{r}_{k}-\bar{r}_{l}\right) \delta\left(r_{k}-R_{0}\right),
$$

where $R_{0}$ is the radius of the nucleus at its surface. The SDI is known to be a reasonable approximation to the effective nucleon-nucleon force in terms of the isoscalar and isovector strengths $a_{0}^{\prime}$ and $a_{1}^{\prime}$. The neutron-proton matrix element of the SDI between harmonic-oscillator states is [5]

$$
\left\langle j_{v} j_{\pi} ; J\left|\hat{V}_{v \pi}^{\mathrm{SDI}}\right| j_{v} j_{\pi} ; J\right\rangle=-\frac{\left(2 j_{v}+1\right)\left(2 j_{\pi}+1\right)}{2}\left[b_{0}\left(\begin{array}{ccc}
j_{v} & j_{\pi} & J \\
\frac{1}{2} & -\frac{1}{2} & 0
\end{array}\right)^{2}+b_{1}\left(\begin{array}{ccc}
j_{v} & j_{\pi} & J \\
-\frac{1}{2} & -\frac{1}{2} & 1
\end{array}\right)^{2}\right],
$$

with

$$
b_{0}=\frac{a_{0}+a_{1}}{2}-(-)^{\ell_{\nu}+\ell_{\pi}+J} \frac{a_{0}-a_{1}}{2}, \quad b_{1}=a_{0},
$$

and $a_{T}=a_{T}^{\prime} C\left(R_{0}\right)$, with $C\left(R_{0}\right)$ and overall scale factor.

The use of the SDI matrix element (3) leads to a geometric understanding of the expression (1) as is illustrated here for $m=m^{\prime}$ with $m=1$ and $m=2$.

\section{The 1-neutron-1-proton matrix elements}

For $m=1$, the expression (1) reduces to the obvious identity $\left\langle j_{v} j_{\pi} ; J\left|\hat{V}_{v \pi}\right| j_{v} j_{\pi} ; J\right\rangle=V_{j_{v} j_{\pi}}^{J}$. Geometric insight in terms of the angle $\theta_{v \pi}$ between the neutron and proton angular-momentum vectors $\bar{J}_{v}$ and $\bar{J}_{\pi}$,

$$
\theta_{v \pi}=\arccos \frac{J(J+1)-j_{v}\left(j_{v}+1\right)-j_{\pi}\left(j_{\pi}+1\right)}{2 \sqrt{j_{v}\left(j_{v}+1\right) j_{\pi}\left(j_{\pi}+1\right)}},
$$


can be obtained following the analysis of Molinari et al. [3], based on Wigner's classical-limit expressions for $3 j$ symbols [6]. The result can be cast in the following form:

$$
\left\langle j_{v} j_{\pi} ; J\left|\hat{V}_{v \pi}^{\mathrm{SDI}}\right| j_{v} j_{\pi} ; J\right\rangle \approx \frac{s_{1}\left(a_{0}, a_{1}\right)}{2 \pi \sin \theta_{v \pi}}+\frac{t_{1}\left(a_{0}, a_{1}\right)}{2 \pi \tan \theta_{v \pi}}
$$

with

$$
\begin{aligned}
& s_{1}\left(a_{0}, a_{1}\right)=-\left(a_{0}+a_{1}\right)+2\left[1+(-)^{j_{\nu}+j_{\pi}+J}\right] a_{0}+(-)^{\ell_{\nu}+\ell_{\pi}+J}\left(a_{0}-a_{1}\right), \\
& t_{1}\left(a_{0}, a_{1}\right)=-(-)^{j_{\nu}+j_{\pi}+J}\left(a_{0}+a_{1}\right)+(-)^{\ell_{\nu}+\ell_{\pi}+j_{\nu}+j_{\pi}}\left(a_{0}-a_{1}\right) .
\end{aligned}
$$

The particle-hole matrix element follows from the expression (1) which leads to the relation

$$
\left\langle j_{v} j_{\pi}^{-1} ; J\left|\hat{V}_{v \pi}\right| j_{v} j_{\pi}^{-1} ; J\right\rangle=-\sum_{R}(2 R+1) V_{j_{v} j_{\pi}}^{R}\left\{\begin{array}{ccc}
j_{v} & j_{\pi} & J \\
j_{v} & j_{\pi} & R
\end{array}\right\} .
$$

This is nothing but the Pandya relation which expresses a particle-hole matrix element as a sum of particle-particle matrix elements [7]. For the SDI, standard angular momentum manipulations lead to the same expression (3) but with

$$
b_{0}=-a_{0}-(-)^{\ell_{\nu}-\ell_{\pi}+J} \frac{a_{0}-a_{1}}{2}, \quad b_{1}=-\frac{a_{0}+a_{1}}{2} .
$$

It is seen that the expression for the particle-hole matrix element of the SDI can be obtained from the particle-particle case with the replacements

$$
a_{0} \mapsto-\frac{a_{0}+a_{1}}{2}, \quad a_{1} \mapsto \frac{a_{1}-3 a_{0}}{2} .
$$

One therefore immediately obtains the classical limit of the particle-hole matrix element of the SDI,

$$
\left\langle j_{v} j_{\pi}^{-1} ; J\left|\hat{V}_{v \pi}^{\mathrm{SDI}}\right| j_{v} j_{\pi}^{-1} ; J\right\rangle \approx \frac{\bar{s}_{1}\left(a_{0}, a_{1}\right)}{2 \pi \sin \theta_{v \pi}}+\frac{\bar{t}_{1}\left(a_{0}, a_{1}\right)}{2 \pi \tan \theta_{v \pi}},
$$

with $\bar{s}_{1}\left(a_{0}, a_{1}\right)=s_{1}\left(-\frac{1}{2}\left(a_{0}+a_{1}\right), \frac{1}{2}\left(a_{1}-3 a_{0}\right)\right)$ and $\bar{t}_{1}\left(a_{0}, a_{1}\right)=t_{1}\left(-\frac{1}{2}\left(a_{0}+a_{1}\right), \frac{1}{2}\left(a_{1}-3 a_{0}\right)\right)$, or explicitly

$$
\begin{aligned}
& \bar{s}_{1}\left(a_{0}, a_{1}\right)=\left[1+(-)^{j_{\nu}+j_{\pi}+J}\right]\left(a_{0}+a_{1}\right)+2 a_{0}+(-)^{\ell_{\nu}+\ell_{\pi}+J}\left(a_{0}-a_{1}\right), \\
& \bar{t}_{1}\left(a_{0}, a_{1}\right)=(-)^{j_{v}+j_{\pi}+J} 2 a_{0}+(-)^{\ell_{\nu}+\ell_{\pi}+j_{v}+j_{\pi}}\left(a_{0}-a_{1}\right) .
\end{aligned}
$$

\section{The 2-neutron-2-proton matrix elements}

For $m=2$, the expression (1) with $\left|J_{v} J_{\pi} ; J\right\rangle \equiv\left|j_{1 v} j_{2 v}\left(J_{v}\right) j_{1 \pi} j_{2 \pi}\left(J_{\pi}\right) J\right\rangle$ reduces to

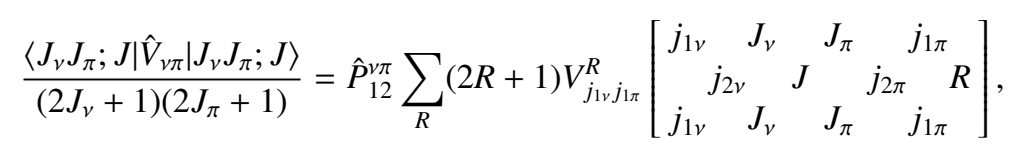

where $\hat{P}_{12}^{v \pi} \equiv \hat{P}_{12}^{v} \hat{P}_{12}^{\pi}$ with $\hat{P}_{12}^{\rho}$ a symmetrizer defined as $\hat{P}_{12}^{\rho} f\left(j_{1 \rho}, j_{2 \rho}\right)=f\left(j_{1 \rho}, j_{2 \rho}\right)+f\left(j_{2 \rho}, j_{1 \rho}\right)$ for any function $f$. The object in square brackets is a $12 j$ symbol of the second kind (see equation (19.2) of Yutsis et al. [8]), a quantity which is scalar under rotations, depending on twelve angular momenta. The corresponding result for 2 neutron-particles and 2 proton-holes is

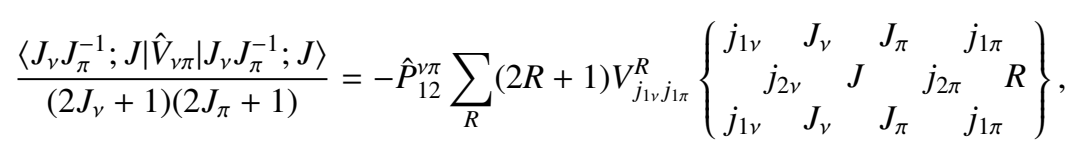


where the object in curly brackets is a $12 j$ symbol of the first kind.

A classical limit can also be obtained for 2-neutron-2-proton matrix element (13). The derivation is more complicated but again makes use of Wigner's classical-limit expressions for $3 j$ symbols [9]. The result can be cast in the form

$$
\left\langle J_{v} J_{\pi} ; J\left|\hat{V}_{v \pi}^{\mathrm{SDI}}\right| J_{v} J_{\pi} ; J\right\rangle \approx \frac{s_{2}\left(a_{0}, a_{1}\right)}{2 \pi \sin \theta_{v \pi}}+\frac{t_{2}\left(a_{0}, a_{1}\right)}{2 \pi \tan \theta_{v \pi}},
$$

where $\theta_{v \pi}$ is now the angle between $\bar{J}_{v}$ and $\bar{J}_{\pi}$, and with

$$
s_{2}\left(a_{0}, a_{1}\right)=-4\left(3 a_{0}+a_{1}\right), \quad t_{2}\left(a_{0}, a_{1}\right)=4 \varphi\left(a_{0}-a_{1}\right), \quad \varphi=\frac{1}{4} \hat{P}_{12}^{v \pi}(-)^{\ell_{1 v}+j_{1 v}+\ell_{1 \pi}+j_{1 \pi}} .
$$

The classical limit of the particle-hole case follows from the replacements (10) and leads to

$$
\left\langle J_{v} J_{\pi}^{-1} ; J\left|\hat{V}_{v \pi}^{\mathrm{SDI}}\right| J_{v} J_{\pi}^{-1} ; J\right\rangle \approx \frac{\bar{s}_{2}\left(a_{0}, a_{1}\right)}{2 \pi \sin \theta_{v \pi}}+\frac{\bar{t}_{2}\left(a_{0}, a_{1}\right)}{2 \pi \tan \theta_{v \pi}} .
$$

Remarkably, one finds $\bar{s}_{2}\left(a_{0}, a_{1}\right)=-s_{2}\left(a_{0}, a_{1}\right)$ and $\bar{t}_{2}\left(a_{0}, a_{1}\right)=t_{2}\left(a_{0}, a_{1}\right)$. The expressions (15) and (17) are accurate only if neutron as well as proton angular momenta are close to aligned, $J_{\rho} \approx j_{1 \rho}+j_{2 \rho}[9]$.

\section{Summary}

The geometric interpretation of the effective nucleon-nucleon interaction can be extended to more complex matrix elements where fixed neutron and proton configurations are coupled to a total angular momentum. The relevant variable is the angle between the neutron and proton angular momenta. The formulas have an identical form in all cases and differ only through their dependence on the strengths in the interaction.

\section{References}

[1] E. Caurier, G. Martínez-Pinedo, F. Nowacki, A. Poves and A.P. Zuker, Rev. Mod. Phys. 77 (2005) 427.

[2] J.P. Schiffer, Ann. Phys. (N.Y.) 66 (1971) 798.

[3] A. Molinari, M. B. Johnson, H. A. Bethe and W. M. Alberico, Nucl. Phys. A 239 (1975) 45.

[4] R. F. Casten, Nuclear Structure from a Simple Perspective (Oxford University Press, Oxford, 2000).

[5] P. J. Brussaard and P. W. M. Glaudemans, Shell-Model Applications in Nuclear Spectroscopy (North-Holland, Amsterdam, 1977).

[6] E. P. Wigner, Group Theory and Its Application to the Quantum Mechanics of Atomic Spectra (Academic Press, New York, 1959).

[7] S. P. Pandya, Phys. Rev. C 103 (1956) 956.

[8] A. P. Yutsis, I. B. Levinson and V. V. Vanagas, The Theory of Angular Momentum (Israel Program for Scientific Translations, Jerusalem, 1962).

[9] P. Van Isacker and A. O. Macchiavelli, Phys. Rev. C 87 (2013) 061301(R). 